\title{
A simple method for measuring plasma power in rf-GDOES instruments
}

\author{
T. Nelis • M. Aeberhard • L. Rohr • J. Michler • \\ P. Belenguer $\cdot$ P. Guillot $\cdot$ L. Thérèse
}

Received: 27 April 2007 / Revised: 11 July 2007 / Accepted: 12 July 2007 / Published online: 4 August 2007

(C) Springer-Verlag 2007

\begin{abstract}
A method for determining plasma power in rfGDOES is presented. It is based on an effective resistance located in the inductive coil of the impedance matching. The amount of electrical power consumed in the matching system depends on the capacitive current flowing through the matching system, which depends on the applied voltage, the stray capacity, and the frequency. This correction method is experimentally evaluated and compared with the integral plasma power calculation.
\end{abstract}

Keywords rf-GDOES $\cdot$ rf-Power control $\cdot$ Blind power

\section{Introduction}

Radio-frequency (rf) powered glow discharges (GD) in the Grimm type configuration are commonly used sources for optical emission [1] and mass spectrometry [2]. The analytical figures of merit of a GD instrument depend strongly on the possibility of controlling the discharge properties. The electrical conditions, voltage and current,

T. Nelis $(\bowtie) \cdot$ M. Aeberhard $\cdot$ L. Rohr $\cdot$ J. Michler

EMPA Materials Science and Technology,

Feurwerkerstrasse 39,

3602 Thun, Switzerland

e-mail: thomas.nelis@empa.ch

P. Belenguer

Laplace, UMR 5213, Université Paul Sabatier,

118 route de Narbonne,

31062 Toulouse cedex, France

P. Guillot $\cdot$ L. Thérèse

Université Jean François Champollion,

Place de Verdun,

81012 Albi cedex, France have a significant effect on sputtering rate [3], emission, and ionisation yields [4]. Variations in the sputtering rate due to changing plasma voltage and current are described by Bouman's equation [3]. The effect of the plasma properties on the emission yield has been discussed in a recent article by Nelis and Bengtson [4]. The emission yields depend on voltage, current, and, to a significantly lesser extent, on the carrier gas pressure and, slightly, on plasma power. Quantification procedures developed for GDOES are based on the constant emission yield approach, requiring either the plasma conditions voltage and current to be constant or the dependence of the emission yield on these conditions to be known and controlled. Both the discharge voltage and current must therefore be carefully controlled for quantitative analysis. Whereas the rf-excitation voltage can be readily measured, the discharge current is difficult to determine, because of the presence of a large capacitive current. Different experimental procedures have been developed to measure $[5,6]$ the discharge current, but they use a complex experimental set-up not always compatible with equipment employed for routine analysis. The method suggested by Wilken at al. [6] uses a current probe surrounding the plasma chamber to directly determine the plasma current. Measuring the electrical power supplied to the plasma " $P_{\mathrm{p}}$ " is a possible replacement for direct current measurement. The plasma power can be estimated from the difference of the rf-power emitted by the generator " $P$ g" and the blind power " $P_{1}$ " consumed by the entire rfsupply circuit including the plasma source without plasma operation. This method was originally suggested by Canpont [7] for GDOES and was later described by Marshall [8]. The method is based on the fact that the blind power increases in proportion to the squared rf-voltage applied to the sample. The proportionality, however, depends strongly on the sample characteristics, in particular its size and shape. 
Although the blind power method has been implemented in many commercially available rf-GDOES systems it has some drawbacks. First, the proportionality factor must be determined for each sample to be analysed. Second the setting of the impedance matching system must be kept constant for the blind power measurement and the actual analysis step. Because the load impedance necessarily changes when the plasma is ignited, the method requires $\mathrm{rf}$ power generators resisting a significant amount of reflected power [8].

The method for determining the "lost" rf-power presented in this work is based on an effective resistance of the rf-circuit. Most parts, for example cables, connectors and capacitors can be made in sufficiently high quality as to avoid measurable power losses. Inductive coils, however, operated at radio frequencies have a quality factor $(Q=\omega L / R)$ of the order of 250 and consequently a significant effective resistance. Payling [9] estimated the effective resistance of the inductive coil used in the automatic impedance matching system ( $\mathrm{L}_{2}$ in Fig. 1) to be on the order of 1 $\mathrm{Ohm}$. The second inductive coil $\left(\mathrm{L}_{1}\right.$ in Fig. 1$)$ used in this experimental set-up is smaller and has, consequently, a smaller effective resistance. Assuming the inductive coil makes the major contribution to power consumption (except for the plasma), the power loss is determined from the electrical current " $\mathrm{L}_{\mathrm{L}}$ " through the inductive coil $\mathrm{L}_{2}$ (Eq. 1)

$P_{1}=P_{\mathrm{g}}-P_{\mathrm{p}}=R_{\mathrm{L}} \times I_{\mathrm{L}}^{2}$

The plasma power can be determined from the difference between the generated power and the "lost" power. In the following discussion we will use the term effective resistance " $R_{\text {eff" }}$ " rather then the equivalent resistance $R_{\mathrm{L}}$

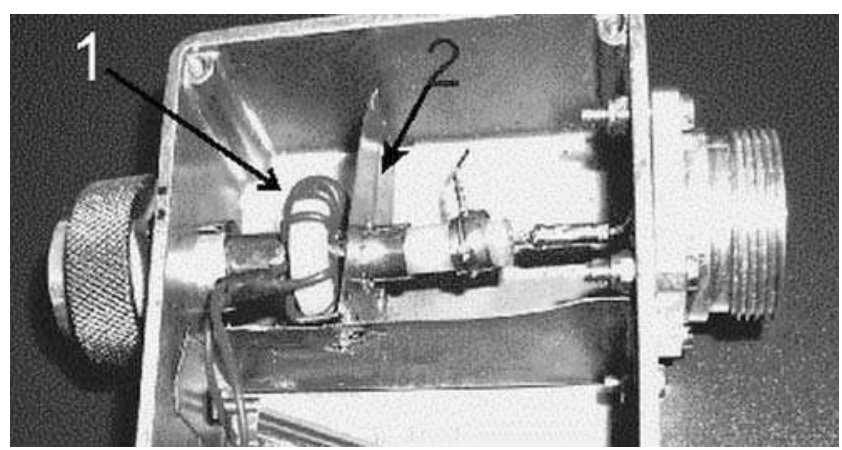

Fig. 2 Photograph of current probe, with coil (1) and electrical shielding (2)

of the inductive coil $\mathrm{L}_{2}$, as other components, not identified in this work, may contribute to the measured power loss and its functional dependence on the electrical current measured at the exit impedance matching system.

\section{Experimental}

All results presented in this work were obtained using a JY 5000 RF GDOES spectrometer (Horiba Jobin Yvon, Longjumeau, France). The layout of the rf supply circuit is displayed in Fig. 1. It has been described in detail previously [9].

A current probe was made in our laboratory using a ferrite ring having a cut off frequency of $40 \mathrm{MHz}$ and an insulated copper wire for the current probe coil (Fig. 2). The voltage generated between the two ends of the coil was used as a measure of the current. Positioned at the exit of the automatic impedance matching system (Point 2 in Fig. 1) and protected from the strong electric field surrounding the rf-power-supply cable, it measures the electrical current flowing through the coil $\mathrm{L}_{2}$.

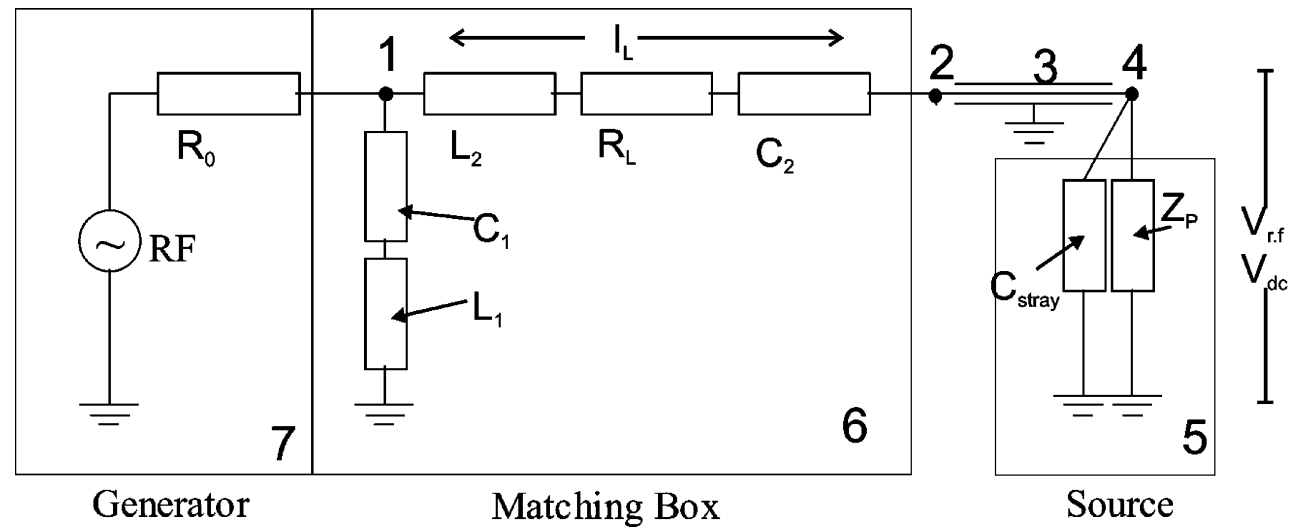

Fig. 1 Layout of the $\mathrm{rf}$ power supply and impedance-matching system: 1 , input of automatic impedance-matching system $(0) ; 2$, exit of automatic impedance; 3, co-axial power supply cable for source (5); $Z_{P}$ represents the plasma impedance, $C_{\text {stray }}$ the stray capacities, $L_{2}$ and $L_{1}$ the inductive coils, and $C_{1}$ and $C_{2}$ the vacuum capacitors included in the automatic matching system; $R_{L}$ represents the effective resistance of the coil $L_{2} ; R_{0}$ is the internal impedance of the rf-power generator (7). $V_{r f f}$ and $V_{d c}$ refer to the potential difference measured between the sample (4) and ground. $I_{L}$ is the current in the inductance $L_{2}$ flowing between points 1 and 2 
Fig. 3 Difference between power for large and small samples as function of squared current. The $A l$ series was measured using an aluminium sample; $\mathrm{Fe} 1$ to $\mathrm{Fe} 3$ series are repeated measurements with the same low-alloy steel sample

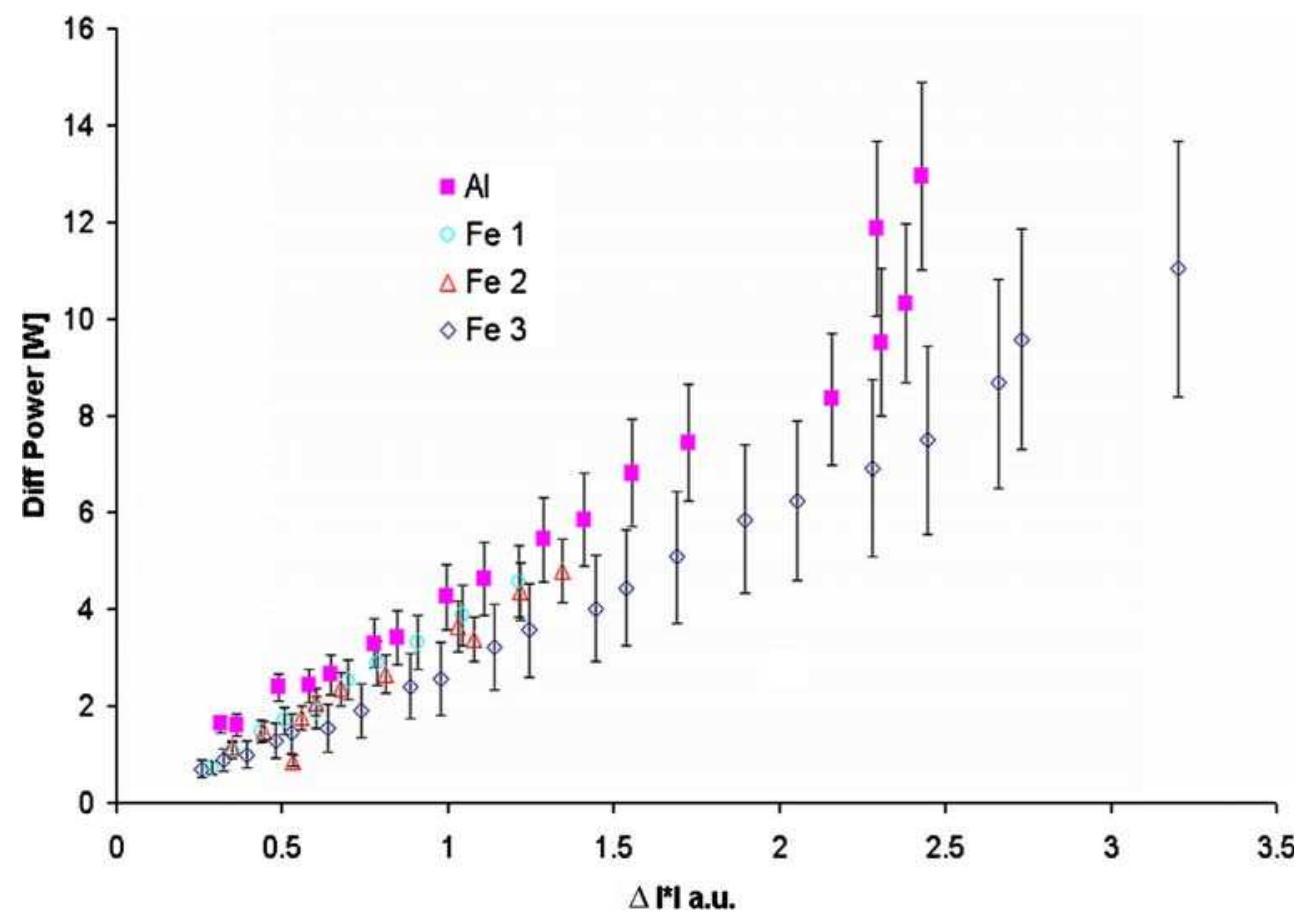

Two different methods were used to verify the assumption that the power loss in the rf-power-supply circuit can be reduced to the effect of one effective resistance.

$I=C_{\text {stray }} \frac{\mathrm{d} V_{\mathrm{rf}}}{\mathrm{d} t}=C_{\text {stray }} \omega V_{\mathrm{rf}}$

The power loss depends on the stray capacity generated by the source, including the sample and the voltage " $V_{\mathrm{rf}}$ " applied to the sample [8]. Equation 2 describes the link between the displacement current, $I_{\mathrm{L}}$, the stray capacity, $C_{\text {stray }}$, and the rf-voltage applied to the sample " $V_{\mathrm{rf}}$ ", including its frequency. Samples of different size and material were used for the experiment. The small samples were discs $2 \mathrm{~cm}$ in diameter and $5 \mathrm{~mm}$ thick. The large samples were exactly the same specimen but backed with a large rectangular $(9 \mathrm{~cm} \times 17 \mathrm{~cm})$ aluminium plate. Low alloyed aluminium and steel were chosen because of their significantly different secondary electron emission yields.

In the first experiment the dc-bias voltage was increased stepwise at constant pressure $(800 \mathrm{~Pa})$ for both small " $P_{\mathrm{gs}}$, $I_{\mathrm{Ls}}$ " and large " $P_{\mathrm{gl}}, I_{\mathrm{Ll}}$ " samples. The forward power delivered by the rf-generator had to be varied from $10 \mathrm{~W}$

Table 1 Equivalent resistance derived from blind power (without plasma ignition) and displacement current measurement for different sample sizes

\begin{tabular}{llll}
\hline$P_{\mathrm{g}}=P_{1}=20 \mathrm{~W}$ & $I_{\text {eff }}$ a.u. & $R_{\text {eff }}$ a.u. & $V_{\text {rf }} \mathrm{rms}(\mathrm{V})$ \\
\hline Small sample & 0.189 & 560 & 660 \\
Large sample & 0.188 & 560 & 512 \\
\hline
\end{tabular}

Table 2 Comparison of plasma power derived

\begin{tabular}{|c|c|c|c|c|}
\hline$P_{\mathrm{g}}(\mathrm{W})$ & $I_{\text {eff }}$ a.u. & $P_{\mathrm{p}}(\mathrm{W})$ & Integral $P_{\mathrm{p}}(\mathrm{W})$ & $V_{\mathrm{dc}}(\mathrm{V})$ \\
\hline \multicolumn{5}{|c|}{ Small sample size } \\
\hline \multicolumn{5}{|c|}{$\mathrm{Al}, 950 \mathrm{~Pa}$} \\
\hline 20 & 0.072 & 17.1 & 17.3 & 318 \\
\hline 30 & 0.08 & 26.4 & 26.2 & 400 \\
\hline 40 & 0.095 & 34.9 & 35 & 460 \\
\hline \multicolumn{5}{|c|}{$\mathrm{Fe}, 550 \mathrm{~Pa}$} \\
\hline 20 & 0.126 & 11.3 & 12.1 & 660 \\
\hline 30 & 0.15 & 17.4 & 18.5 & 800 \\
\hline 40 & 0.173 & 23 & 24.2 & 920 \\
\hline \multicolumn{5}{|c|}{$\mathrm{Fe}, 700 \mathrm{~Pa}$} \\
\hline 20 & 0.107 & 13.6 & 14.2 & 540 \\
\hline 30 & 0.129 & 20.7 & 21.5 & 870 \\
\hline 40 & 0.151 & 27.2 & 28.5 & 920 \\
\hline \multicolumn{5}{|c|}{$\mathrm{Fe} 950 \mathrm{~Pa}$} \\
\hline 20 & 0.083 & 16.1 & 16.4 & 380 \\
\hline 30 & 0.1 & 24.4 & 24.8 & 480 \\
\hline 40 & 0.117 & 32.3 & 33.1 & 550 \\
\hline \multicolumn{5}{|c|}{ Large sample size } \\
\hline \multicolumn{5}{|c|}{$\mathrm{Fe} 550 \mathrm{~Pa}$} \\
\hline 20 & 0.144 & 8.4 & 8.17 & 582 \\
\hline 30 & 0.173 & 13.2 & 13.1 & 711 \\
\hline 40 & 0.201 & 17.4 & 17.2 & 806 \\
\hline \multicolumn{5}{|c|}{$\mathrm{Fe} 700 \mathrm{~Pa}$} \\
\hline 20 & 0.126 & 11.2 & 11 & 501 \\
\hline 30 & 0.151 & 17.2 & 17.4 & 620 \\
\hline 40 & 0.176 & 22.7 & 22.5 & 705 \\
\hline \multicolumn{5}{|c|}{$\mathrm{Fe} 950 \mathrm{~Pa}$} \\
\hline 20 & 0.099 & 14.5 & 14.3 & 360 \\
\hline 30 & 0.12 & 21.9 & 21.8 & 460 \\
\hline 40 & 0.139 & 29.2 & 29 & 530 \\
\hline
\end{tabular}


Fig. 4 Intensity-time profiles measured at $40 \mathrm{~W}$ and $700 \mathrm{~Pa}$ for samples of a computer hard disc of different sizes. The results for the small piece (squares) are identified by higher intensities and faster rates of erosion. The upper trace displays the effect of sample size on the auto-bias voltage, in V. Small samples result in a higher auto-bias voltage
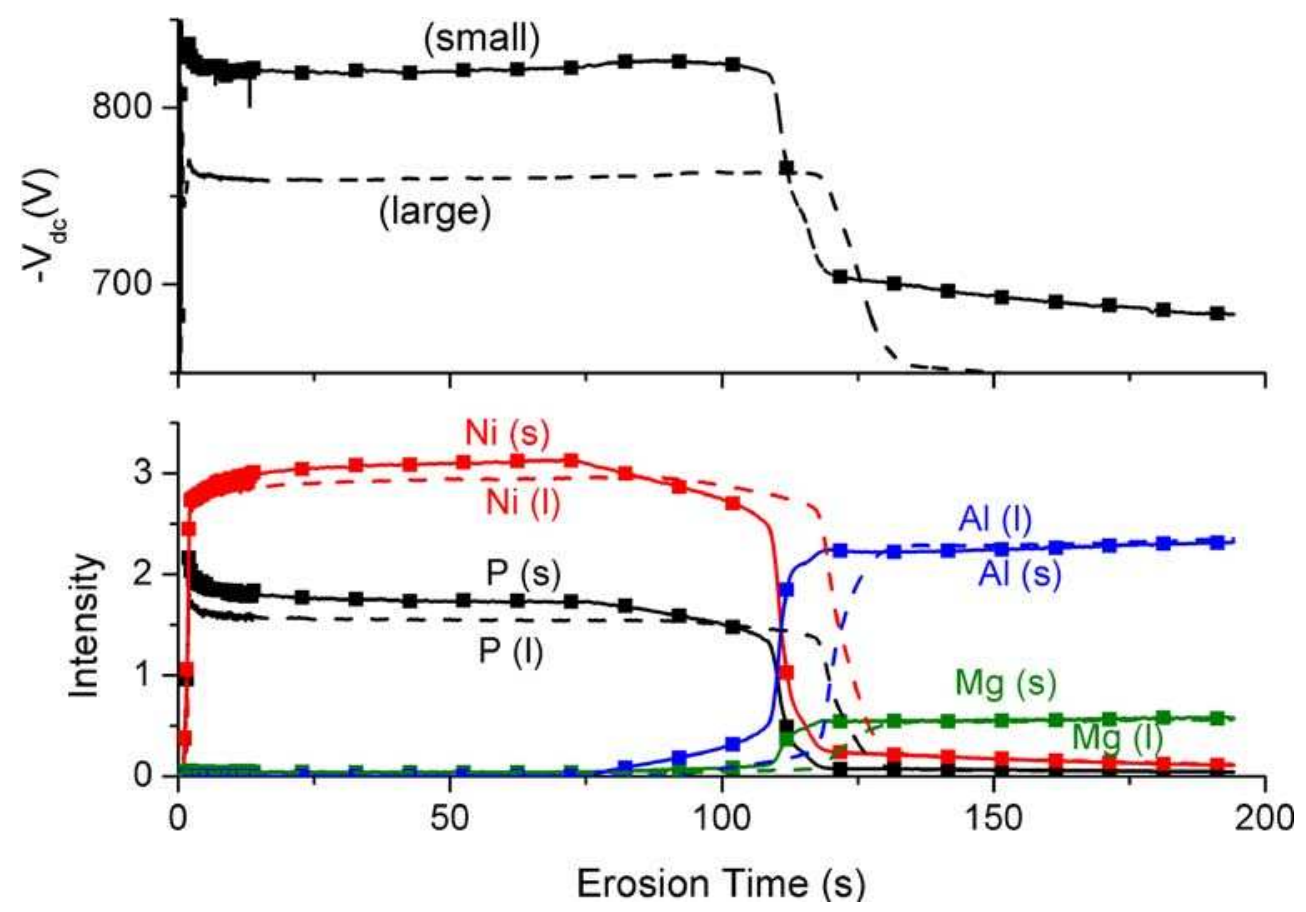

to $90 \mathrm{~W}$ during the experiment. For each $V_{\mathrm{dc}}$ setting the difference in required generator power was related to the difference in the square of the current probe signal (Eq. 3), because for a given cathode material, chamber geometry, and constant plasma carrier gas pressure (density), the plasma power is a function of the discharge voltage only.

$$
\Delta P_{1}=P_{\mathrm{gl}}-P_{\mathrm{gs}}=R_{\mathrm{eff}} \times\left(I_{\mathrm{LI}}^{2}-I_{\mathrm{Ls}}^{2}\right)
$$

The results are displayed in Fig. 3. The measurement uncertainty was estimated to be $1.5 \%$ of the generator power reading. The measurement uncertainty is because of both the accuracy of power reading and the spot-to-spot reproducibility of the discharge conditions. The difference in slope observed for the different sample materials is therefore within the limit of experimental uncertainty. The experimental results confirm the assumption of a single effective resistance. The power loss increases linearly with the square of the capacitive current. The effect of sample size on power loss, and its dependence on the excitation voltage, are independent of the sample material, i.e. the source impedance.

This first experiment is close to routine analytical application and supports the assumption of the effective resistance being responsible for the major part of the lost power in GDOES. We nevertheless checked the assumption using a different approach.

In this second experiment we compared the effective resistance approach with the integral method suggested by Belenguer [5]. The effective resistance was calculated from blind power and capacitive current measurements without plasma ignition. The experimental results are summarised in Table 1.
Whereas the rf-voltage at the sample varies strongly with sample size, the capacitive current measured by the current probe (Fig. 2) is independent of the sample. This implies that the power consumption is directly linked to the capacitive current " $I_{\mathrm{L}}$ ", but only indirectly, via the variable stray capacities, to the potential difference across the discharge

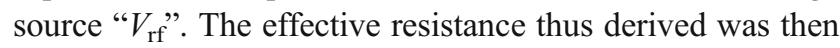
used to derive the plasma power for a large range of discharge conditions. A comparison of the results with integral power measurements is shown in Table 2. All measurements were performed in the automatic impedance matching mode minimising the reflected power to a negligible value.

The values derived by both methods are in good agreement, within $1 \mathrm{~W}$, independent of sample size, total rf power, and source impedance.

\section{Analytical relevance}

To illustrate the relevance of the power transmission to analytical routine work we analysed a computer hard disc,

Table 3 Line intensities on NiP layer of computer hard disk, on a small sample and on the full hard disk

\begin{tabular}{llllll}
\hline HDD Size & Set $P_{\mathrm{f}}(\mathrm{W})$ & $P_{\mathrm{p}}(\mathrm{W})$ & $\mathrm{P}(\mathrm{au})$ & $\mathrm{Ni}(\mathrm{au})$ & $V_{\mathrm{dc}}(\mathrm{V})$ \\
\hline Small & 40 & 27.9 & 1.94 & 3.3 & 815 \\
Full & 40 & 25.8 & 1.75 & 2.9 & 760 \\
Full & 44 & 28.3 & 1.93 & 3.2 & 811 \\
\hline
\end{tabular}

$P_{\mathrm{f}}$ is the forward power delivered by the generator; $P_{\mathrm{p}}$ is the plasma power derived from the capacitive current 
first as a whole piece, a disc $9 \mathrm{~cm}$ in diameter and then as a small sample $(1 \mathrm{~cm} \times 2 \mathrm{~cm})$ cut from the same specimen. These hard discs are often used as test samples in GDOES because they have a very reproducible layer structure and homogeneous elemental composition [10]. The nickel phosphate layer and the aluminium-magnesium alloy base material have significantly different secondary electron emission yields, leading to different source impedance. The intensity-time profiles are compared in Fig. 4.

The small sample results in higher intensities and faster erosion. The dc bias voltage decreases for both samples from the NiP layer to the aluminium alloy base material. The average voltage is significantly higher for the small sample. The difference between the detected line intensities is much stronger for the NiP layer and rather small for the aluminium alloy. Increasing the generator power from $40 \mathrm{~W}$ to $44 \mathrm{~W}$ for the large sample is sufficient to compensate for the reduced power transmission. Table 3 shows the emitted average intensities and dc bias voltages for the three configurations; each value is derived from an average of four consecutive measurements. A general calibration procedure, based on the constant emission-yield approach, will necessary fail to supply accurate results. Both, a $V_{\mathrm{dc}}$ correction or pressure regulation, used to compensate for the effects of changes in the source caused by the varying secondary electron emission yield must fail, because the reason for the different auto-bias voltage is not the composition of the sample but its size. An analytical result depending on sample size is clearly not satisfactory.

\section{Discussion}

This work demonstrates that the power losses typically observed in rf-GDOES are caused by the non-negligible resistance of the inductive coil, which is part of the impedance-matching system. The amount of electrical power consumed in the matching system depends on the capacitive current flowing through the matching system. The capacitive current depends on the excitation voltage and the stray capacity, which in turn depends on sample shape and size. The analytical results obtained by rfGDOES, therefore depend on the sample shape. Uncontrolled variation of the plasma power directly affects the intensity-time profiles and renders control and/or correction of the dependence of emission and ionisation yields impossible and, consequently, significantly reduces the accuracy of the results obtained.

Two independent experimental proofs show that the resistance of the impedance matching system, i.e. the inductive coil, is the dominant source of power losses. Measurement of the capacitive current enables real-time determination of the power losses and, consequently, calculation of the plasma power.

This correction method is independent of the sample characteristics, the discharge conditions, and the impedance matching settings. It is therefore compatible with automatic impedance matching operation.

In future work it is planned to integrate effective resistance correction into the spectrometer, enabling its use in routine analysis. We finally plan to verify the affect on analysis of non-conducting samples, for which power losses are even more important, because of the higher excitation voltages [11] required to obtain suitable discharge conditions.

The correction method is compatible with most commercially available rf-GDOES and MS instruments and can easily be integrated into existing systems.

\section{References}

1. Nelis Th, Payling R (2004) A guidebook to glow discharge optical emission spectroscopy. RSC

2. Hohl M, Kanzari A, Michler J, Nelis Th, Fuhrer K, Gonin M (2006) Surf Interface Anal 38:292-295

3. Payling R, Aeberhard M, Michler J, Authier C, Chapon P, Nelis Th, Pitchford L (2003) Surf Interface Anal 35:334-339

4. Bengtson A, Nelis Th (2006) Anal Bioanal Chem 385:568-585

5. Belenguer $\mathrm{Ph}$, Guillot $\mathrm{Ph}$, Therèse L (2003) Surf Interface Anal 35:604-610

6. Wilken L, Hoffmann V, Uhlemann H-J, Siegel H, Wetzig K (2003) J Anal At Spectrom 18:646-655

7. Canpont F (1993) PhD Thesis, Universitè Claude Bernard, Lyon

8. Marshall KA, Casper TJ, Brushwyler KR, Mitchell JC (2003) J Anal At Spectrom 18:637-645

9. Payling R, Bonnot O, Fretel E, Rogerieux O, Aeberhard M, Michler J, Nelis Th, Hansen U, Hartmann A, Belenguer Ph, Guillot Ph (2003) J Anal At Spectrom 18:656-664

10. Nelis Th, Pallosi J (2006) Appl Spectrosc Rev 41:227-258

11. Therese L, Ghalem Z, Guillot P, Belenguer P (2006) Anal Bioanal Chem 386:163-168 